\title{
Shape Modification for $\lambda$-Bézier Curves Based on Constrained Optimization of Position and Tangent Vector
}

\author{
Gang Hu, ${ }^{1}$ Xiaomin Ji, ${ }^{2}$ Xinqiang Qin, ${ }^{1}$ and Suxia Zhang ${ }^{1}$ \\ ${ }^{1}$ Department of Applied Mathematics, Xian University of Technology, Xian 710054, China \\ ${ }^{2}$ College of the Arts, Xian University of Technology, Xian 710048, China \\ Correspondence should be addressed to Gang Hu; peng_gh@163.com
}

Received 7 October 2014; Revised 15 January 2015; Accepted 23 January 2015

Academic Editor: Chih-Cheng Hung

Copyright (c) 2015 Gang Hu et al. This is an open access article distributed under the Creative Commons Attribution License, which permits unrestricted use, distribution, and reproduction in any medium, provided the original work is properly cited.

\begin{abstract}
Besides inheriting the properties of classical Bézier curves of degree $n$, the corresponding $\lambda$-Bézier curves have a good performance on adjusting their shapes by changing shape control parameter. Specially, in the case where the shape control parameter equals zero, the $\lambda$-Bézier curves degenerate to the classical Bézier curves. In this paper, the shape modification of $\lambda$-Bézier curves by constrained optimization of position and tangent vector is investigated. The definition and properties of $\lambda$-Bézier curves are given in detail, and the shape modification is implemented by optimizing perturbations of control points. At the same time, the explicit formulas of modifying control points and shape parameter are obtained by Lagrange multiplier method. Using this algorithm, $\lambda$-Bézier curves are modified to satisfy the specified constraints of position and tangent vector, meanwhile the shape-preserving property is still retained. In order to illustrate its ability on adjusting the shape of $\lambda$-Bézier curves, some curve design applications are discussed, which show that the proposed method is effective and easy to implement.
\end{abstract}

\section{Introduction}

Bézier curves are widely used in Computer Aided Geometric Design (CAGD) and computer graphics (CG) which have many properties that are helpful for shape design. Developing more convenient techniques for designing and modifying Bézier curves is an important problem in computer aided design (CAD), computer aided manufacturing (CAM), and NC technology fields; see [1]. However, shape design is timeconsuming and usually cannot be accomplished in one stroke. After creating Bézier curves or surfaces, we often need to modify them so that their shapes can satisfy our design requirements.

Many efforts have been made to develop more convenient and effective methods for shape modification of parametric curves and surfaces. Piegl [2] proposed two methods to alter the shape of NURBS curves, including control-pointbased modification and weight-based modification. SànchezReyes [3] developed a simple technique to modify NURBS curves based on a perspective functional transformation of arbitrary origin. Juhász [4] provided a weight-based shape modification method, by which one can prescribe not only the new position of an arbitrary chosen point of a plane NURBS curves but the tangent direction as well. $\mathrm{Hu}$ et al. $[5,6]$ developed a method for shape modification of NURBS curves and surfaces with geometric constraint. However, developing more effective way for shape modification of Bézier curves is still an important problem. Inspired by the results in [5, 6], Xu et al. [7] proposed a method to modify the shape of Bézier curves by minimizing the changes of the shape by least square, where explicit formulas are deduced to calculate positions of new control points of the modified curve. Wu and Xia [8] investigated the optimal shape modification of Bézier curves by geometric constraint, where shape modification of Bézier curve with added end-point and tangent constraints is discussed. Wang et al. [9] presented a method for shape modification of NURBS curves, which is based on constrained optimization by means of altering the corresponding weights of their control points. Juhász and Hoffmann [10] investigated the effect of the modification of knot values on the shape of B-spline curves. Han and Ren [11] investigated geometric constrained optimization for shape modification of Bézier curves and obtained precise formula for it. 
Bézier curves have now become a powerful tool for constructing free-form curves in CAD/CAM. However, the Bézier model is imperfect, and it has its own shortage. That is, after choosing the basis functions, the shape of a Bézier curve is well determined by its control points. In the recent years, in order to overcome the shortage of Bézier curves, many scholars constructed new curves whose structures are similar to the Bézier curves by introducing parameters into basis functions; see [12-21]. These new curves share many basic properties with the Bézier curves and at the same time hold flexible shape adjustable property. Yan and Liang [16] constructed a new kind of basis functions by a recursive approach and defined a kind of parametric curves called $\lambda$-Bézier curves with shape parameter based on these basis functions. The new curves have most properties of the corresponding classical Bézier curves. Moreover, the shape parameter can adjust the shape of the new curves without changing the control points. Focusing on the problem of shape modification of $\lambda$-Bézier curves, we study the shape modification of $\lambda$-Bézier curves by constrained optimization of position and tangent vector and obtain the explicit formulas of the modified control points and shape parameter.

The remainder of the paper is organized as follows. The definition and properties of $\lambda$-Bézier curves are given in Section 2. In Section 3, we present the shape modification of $\lambda$-Bézier curve by constrained optimization of single point constraint. Practical examples are given in Section 4, and we present some applications. At last, a short conclusion is given in Section 5.

\section{The Definition and Properties of $\lambda$-Bézier Curves}

2.1. Extension Basis Function. The definition of extension Bernstein basis functions is given as follows [16].

Definition 1. Let $\lambda \in[-1,1]$; for $t \in[0,1]$, the following polynomial functions

$$
\begin{aligned}
& b_{0,2}(t ; \lambda)=\left(1-2 \lambda t+\lambda t^{2}\right)(1-t)^{2}, \\
& b_{1,2}(t ; \lambda)=2 t(1-t)\left(1+\lambda-\lambda t+\lambda t^{2}\right), \\
& b_{2,2}(t ; \lambda)=\left(1-\lambda+\lambda t^{2}\right) t^{2}
\end{aligned}
$$

$$
t \in[0,1]
$$

are called the extension Bernstein basis functions of degree 2 associated with the shape parameter $\lambda$.

For any integer $n(n \geq 3)$, the functions $b_{i, n}(t ; \lambda)(i=$ $0,1, \ldots, n)$, defined recursively by

$$
\begin{aligned}
b_{i, n}(t ; \lambda)=(1-t) b_{i, n-1}(t ; \lambda)+t b_{i-1, n-1}(t ; \lambda), & \\
& t \in[0,1]
\end{aligned}
$$

are called the extension Bernstein basis functions of degree $n$. In the case $k=-1$ or $k>l$, we set $b_{k, l}(t ; \lambda)=0$.
Theorem 2. The extension Bernstein basis functions of degree $n$ can be expressed explicitly as

$$
\begin{aligned}
& b_{i, n}(t ; \lambda) \\
& \quad=\left(1+\frac{3 C_{n-2}^{i-1}+C_{n-1}^{i}-C_{n}^{i}}{C_{n}^{i}} \lambda-\frac{2 C_{n-1}^{i}}{C_{n}^{i}} \lambda t+\lambda t^{2}\right) \\
& \cdot C_{n}^{i} t^{i}(1-t)^{n-1} \quad(i=0,1, \ldots, n),
\end{aligned}
$$

where $n \geq 2, C_{n}^{i}=n ! / i !(n-i) !$.

The extension Bernstein basis functions (3) have the following properties:

(a) Degeneracy. In the particular case where the shape control parameter $\lambda$ equals zero, the extension Bernstein basis functions of degree $n$ are just the classical ones of the same degree.

(b) Nonnegativity. For any $\lambda \in[-1,1], b_{i, n}(t ; \lambda) \geq 0(i=$ $0,1, \ldots, n)$, where $n \geq 2$.

(c) Partition of Unity. One has $\sum_{i=0}^{n} b_{i, n}(t ; \lambda)=1$.

(d) Symmetry. One has $b_{i, n}(1-t ; \lambda)=b_{n-i, n}(t ; \lambda)(i=$ $0,1, \ldots, n)$.

(e) Linear Independence. For any $\lambda \in[-1,1]$, the extension Bernstein basis functions $b_{i, n}(t ; \lambda)(i=$ $0,1, \ldots, n)$ are linearly independent.

\subsection{Construction of $\lambda$-Bézier Curves}

Definition 3. Given control points $\mathbf{P}_{i}(i=0,1, \ldots, n ; n \geq 2)$ in $R^{2}$ or $R^{3}$, then

$$
\mathbf{p}(t ; \lambda)=\sum_{i=0}^{n} \mathbf{P}_{i} b_{i, n}(t ; \lambda), \quad t \in[0,1], \lambda \in[-1,1]
$$

is called a $\lambda$-Bézier curve of degree $n$ with shape parameter $\lambda$, where basis functions $b_{i, n}(t ; \lambda)(i=0,1, \ldots, n ; n \geq 2)$ are defined by (3) (see Definition 3.2 in [16]).

From the properties of the extension Bernstein basis functions (3), the $\lambda$-Bézier curves (4) have the following properties.

(a) End-Point Properties. For any $\lambda \in[-1,1]$, we have

$$
\begin{aligned}
& \mathbf{p}(0 ; \lambda)=\mathbf{P}_{0}, \\
& \mathbf{p}(1 ; \lambda)=\mathbf{P}_{n} .
\end{aligned}
$$

And the derivative at end-points will satisfy

$$
\begin{aligned}
& \mathbf{p}^{\prime}(0 ; \lambda)=(n+2 \lambda)\left(\mathbf{P}_{1}-\mathbf{P}_{0}\right) \\
& \mathbf{p}^{\prime}(1 ; \lambda)=(n+2 \lambda)\left(\mathbf{P}_{n}-\mathbf{P}_{n-1}\right) .
\end{aligned}
$$


Furthermore, the second derivative at end-points can be represented as

$$
\begin{aligned}
\mathbf{p}^{\prime \prime}(0 ; \lambda)= & {\left[2 \lambda+(4 \lambda-1) n+n^{2}\right] \mathbf{P}_{0} } \\
& +\left[8 \lambda+(2-8 \lambda) n-2 n^{2}\right] \mathbf{P}_{1} \\
& +\left[-10 \lambda+(4 \lambda-1) n+n^{2}\right] \mathbf{P}_{2}, \\
\mathbf{p}^{\prime \prime}(1 ; \lambda)= & {\left[-10 \lambda+(4 \lambda-1) n+n^{2}\right] \mathbf{P}_{n-2} } \\
& +\left[8 \lambda+(2-8 \lambda) n-2 n^{2}\right] \mathbf{P}_{n-1} \\
& +\left[2 \lambda+(4 \lambda-1) n+n^{2}\right] \mathbf{P}_{n} .
\end{aligned}
$$

(b) Symmetry. Considering $\mathbf{P}_{0}, \mathbf{P}_{1}, \ldots, \mathbf{P}_{n}(n \geq 2)$ and $\mathbf{P}_{n}, \mathbf{P}_{n-1}, \ldots, \mathbf{P}_{0}(n \geq 2)$, define the same $\lambda$-Bézier curves; that is,

$$
\mathbf{p}\left(t ; \lambda ; \mathbf{P}_{0}, \mathbf{P}_{1}, \ldots, \mathbf{P}_{n}\right)=\mathbf{p}\left(1-t ; \lambda ; \mathbf{P}_{n}, \mathbf{P}_{n-1}, \ldots, \mathbf{P}_{0}\right) .
$$

(c) Convex Hull Property. The entire $\lambda$-Bézier curves must lie inside the convex hull of its control polygon spanned by $\mathbf{P}_{0}, \mathbf{P}_{1}, \ldots, \mathbf{P}_{n}(n \geq 2)$.

(d) Affine Invariance. The shape of $\lambda$-Bézier curve is independent of the choice of coordinates; that is, (4) satisfies the following two equations:

$$
\begin{aligned}
& \mathbf{p}\left(t ; \lambda ; \mathbf{P}_{0}+\mathbf{Q}, \mathbf{P}_{1}+\mathbf{Q}, \ldots, \mathbf{P}_{n}+\mathbf{Q}\right) \\
& \quad=\mathbf{p}\left(t ; \lambda ; \mathbf{P}_{0}, \mathbf{P}_{1}, \ldots, \mathbf{P}_{n}\right)+\mathbf{Q} \\
& \mathbf{p}\left(t ; \lambda ; \mathbf{M P} \mathbf{P}_{0}, \mathbf{M} \mathbf{P}_{1}, \ldots, \mathbf{M} \mathbf{P}_{n}\right) \\
& \quad=\mathbf{M p}\left(t ; \lambda ; \mathbf{P}_{0}, \mathbf{P}_{1}, \ldots, \mathbf{P}_{n}\right),
\end{aligned}
$$

where $\mathbf{Q}$ is an arbitrary vector in $R^{2}$ or $R^{3}$ and $\mathbf{M}$ is an arbitrary $l \times l$ matrix, $l=2$ or 3 .

(e) Variation Diminishing Property. Since the extension Bernstein basis functions given in (3) form a group of (optimal) normalized totally positive basis functions, the corresponding $\lambda$-Bézier curves possess variation diminishing property, which means that no plane intersects $\lambda$-Bézier curve more often than it intersects the corresponding control polygon.

\subsection{Performance Comparison of $\lambda$-Bézier Curves, Bézier} Curves, and NURBS. A Bézier curve is defined as a parametric one which forms the basis of the Bernstein function. However, once the control points and their corresponding Bernstein polynomials are given, the shape of a Bézier curve is formed uniquely and there is no possibility to adjust it anymore. Modifying the shape of Bézier curves essentially requires the adjustments of vertexes of the control polygon, which is very inconvenient. For these reasons, the problem of shape modification of curves is proposed. Although the
TABle 1: Performance comparisons of $\lambda$-Bézier curves, Bézier curves, and NURBS.

\begin{tabular}{lccc}
\hline Property & $\begin{array}{c}\lambda \text {-Bézier } \\
\text { curves }\end{array}$ & $\begin{array}{c}\text { Bézier } \\
\text { curves }\end{array}$ & $\begin{array}{c}\text { NURBS } \\
\text { curves }\end{array}$ \\
\hline Property of basis functions & $\checkmark$ & & \\
Nonnegativity & $\checkmark$ & $\checkmark$ & $\checkmark$ \\
Partition of unity & $\checkmark$ & $\checkmark$ & $\checkmark$ \\
Symmetry & $\checkmark$ & $x$ & $*$ \\
Shape parameters & $\checkmark$ & $\checkmark$ & $\checkmark$ \\
Linear independence & $\checkmark$ & $x$ & $\checkmark$ \\
Degeneracy & & & \\
Property of the Curves & $\checkmark$ & $\checkmark$ & $\checkmark$ \\
Variation diminishing property & $\checkmark$ & $\checkmark$ & $\checkmark$ \\
Affine invariability & $\checkmark$ & $\checkmark$ & $\checkmark$ \\
Convex hull property & $\checkmark$ & $\checkmark$ & $\checkmark$ \\
Symmetry & $\checkmark$ & $\checkmark$ & $\checkmark$ \\
End-point properties & $\checkmark$ & $x$ & $\checkmark$ \\
Extra degree of freedom & Low & Low & High \\
Computational complexity & & & \\
\hline
\end{tabular}

${ }^{*}$ The weights in NURBS methods possess an effect for adjusting the shape of the curves.

weights in NURBS method can adjust the shapes of NURBS curve and the NURBS curve has good properties and can express the conic section, the NURBS curve also has disadvantages, such as difficulty in choosing the value of the weight, the increased order of rational fraction caused by the derivation, and the need for a numerical method of integration.

The shape parameters are applied to generate some curves whose shape is adjustable as an extension of the existing method. The $\lambda$-Bézier curves (4) have most properties of the corresponding classical Bézier curves. Moreover, the shape parameter can adjust the shape of the $\lambda$-Bézier curves without changing the control points. With the increasing of the shape parameter, the $\lambda$-Bézier curves approach to the control polygon or control net, and the $\lambda$-Bézier model can approximate the control polygon or control net better than the classical Bézier model. In addition, the expressions of $\lambda$ Bézier curves defined in this paper are more concise compared with the Bézier curves and NURBS curves. Particularly, when the shape parameter $\lambda$ equals zero, the $\lambda$-Bézier curves (4) degenerate to the classicalBézier curves.

To sum up, with the extra degree of freedom provided by the shape parameter $\lambda$ in $b_{i, n}(t ; \lambda) \geq 0(i=0,1, \ldots, n)$, the curves $\mathbf{p}(t ; \lambda)$ can be freely adjusted and controlled by changing the value of $\lambda$ instead of changing the control points $\mathbf{P}_{0}, \mathbf{P}_{1}, \ldots, \mathbf{P}_{n}$. Performances of $\lambda$-Bézier curves, Bézier curves, and NURBS curves are compared in detail in Table 1.

Figure 1 shows graphs of $\lambda$-Bézier curves with the same control polygon but different shape parameters. Figure 1(a) shows the curves generated by the extension Bernstein basis functions with $n=4$ and $\mathbf{p}(t ; 1)$ (solid lines), $\mathbf{p}(t ; 0)$ (dashed lines), and $\mathbf{p}(t ;-1)$ (dot-dashed lines), respectively. Figure $1(\mathrm{~b})$ shows the curves generated by the extension 


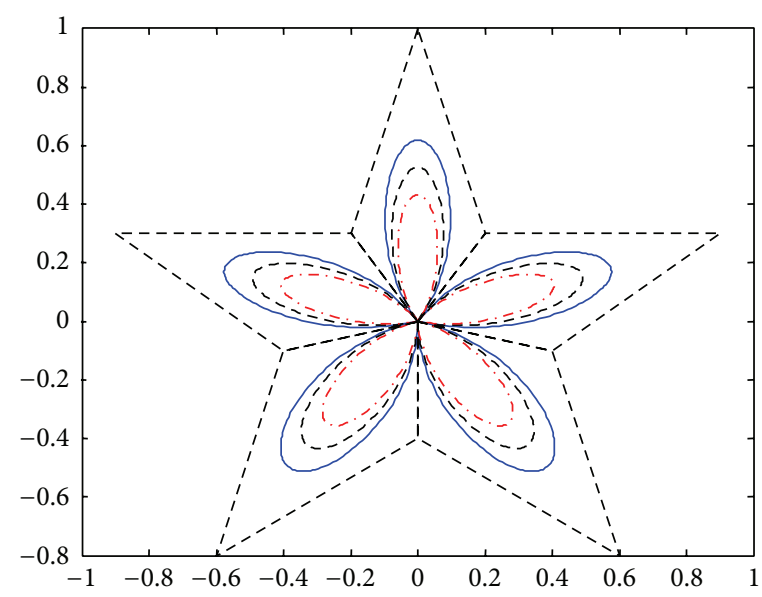

(a) $\lambda$-Bézier curves of degree 4

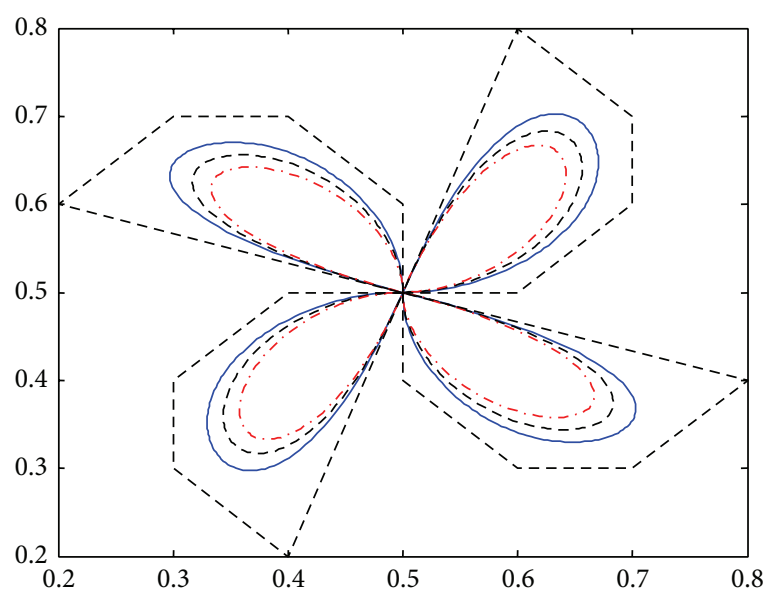

(b) $\lambda$-Bézier curves of degree 5

FIGURE 1: $\lambda$-Bézier curves with the same control polygon but different shape parameters.

Bernstein basis functions with $n=5$ and $\mathbf{p}(t ; 1)$ (solid lines), $\mathbf{p}(t ; 0)$ (dashed lines), and $\mathbf{p}(t ;-1)$ (dot-dashed lines), respectively. From the figures, we can see that the $\lambda$-Bézier curves approach to the control polygon when the shape parameter is increased.

\section{Shape Modification for $\lambda$-Bézier Curves by Constrained Optimization}

$\lambda$-Bézier curve of degree $n$ with control points $\mathbf{P}_{0}, \mathbf{P}_{1}, \ldots$, $\mathbf{P}_{n}(n \geq 2)$ is

$$
\mathbf{p}_{1}\left(t ; \lambda_{1}\right)=\sum_{i=0}^{n} \mathbf{P}_{i} b_{i, n}\left(t ; \lambda_{1}\right), \quad t \in[0,1] .
$$

Modified curves are

$$
\begin{aligned}
\mathbf{p}_{2}\left(t ; \lambda_{1}\right)= & \sum_{i=0}^{l-1} \mathbf{P}_{i} b_{i, n}\left(t ; \lambda_{1}\right)+\sum_{i=l}^{m}\left(\mathbf{P}_{i}+\boldsymbol{\delta}_{i}\right) b_{i, n}\left(t ; \lambda_{1}\right) \\
& +\sum_{i=m+1}^{n} \mathbf{P}_{i} b_{i, n}\left(t ; \lambda_{1}\right), \\
\mathbf{p}_{3}\left(t ; \lambda_{2}\right)= & \sum_{i=0}^{l-1} \mathbf{P}_{i} b_{i, n}\left(t ; \lambda_{2}\right)+\sum_{i=l}^{m}\left(\mathbf{P}_{i}+\boldsymbol{\delta}_{i}\right) b_{i, n}\left(t ; \lambda_{2}\right) \\
& +\sum_{i=m+1}^{n} \mathbf{P}_{i} b_{i, n}\left(t ; \lambda_{2}\right) .
\end{aligned}
$$

Here, $\boldsymbol{\delta}_{i}=\left(\delta_{i}^{x}, \delta_{i}^{y}, \delta_{i}^{z}\right)^{T}(i=l, l+1, \ldots, m)$ are perturbations of control points $\mathbf{P}_{i}(i=l, l+1, \ldots, m)$.

3.1. Position Vector Constraint. Suppose the position vector of $\lambda$-Bézier curves (10) is $\mathbf{p}_{1}\left(t^{*} ; \lambda_{1}\right)=\mathbf{S}_{1}$ and then that of modified curve (11) satisfies $\mathbf{p}_{2}\left(t^{*} ; \lambda_{1}\right)=\mathbf{S}_{2}$; here

$$
\mathbf{p}_{2}\left(t^{*} ; \lambda_{1}\right)=\mathbf{S}_{1}+\sum_{i=l}^{m} \boldsymbol{\delta}_{i} b_{i, n}\left(t^{*} ; \lambda_{1}\right)=\mathbf{S}_{2} .
$$

For $n \geq 2$ and $0 \leq l \leq m \leq n$, perturbation $\boldsymbol{\delta}_{i}$ can be computed by constrained optimization with $\sum_{i=l}^{m}\left\|\boldsymbol{\delta}_{i}\right\|^{2}$ being minimum. The Lagrange function is defined as follows:

$$
L=\sum_{i=l}^{m}\left\|\boldsymbol{\delta}_{i}\right\|^{2}+\boldsymbol{\alpha}^{T}\left[\mathbf{S}_{2}-\mathbf{p}_{2}\left(t^{*} ; \lambda_{1}\right)\right],
$$

where $\boldsymbol{\alpha}^{T}=\left(\alpha^{x}, \alpha^{y}, \alpha^{z}\right)$ is the Lagrange multiplier and $\|\cdot\|$ is Euclidean norm.

Theorem 4. When $t^{*} \in(0,1)$ and $n \geq 2$, then (14) has a unique solution.

Proof. Let perturbation $\boldsymbol{\delta}_{i}=\left(\delta_{i}^{x}, \delta_{i}^{y}, \delta_{i}^{z}\right)^{T}$ satisfy

$$
\begin{aligned}
\frac{\partial L}{\partial \delta_{i}^{x}} & =\frac{\partial L}{\partial \delta_{i}^{y}}=\frac{\partial L}{\partial \delta_{i}^{z}}=0, \quad i=l, l+1, \ldots, m \\
\frac{\partial L}{\partial \alpha^{x}} & =\frac{\partial L}{\partial \alpha^{y}}=\frac{\partial L}{\partial \alpha^{z}}=0 .
\end{aligned}
$$

We can obtain

$$
\begin{aligned}
\boldsymbol{\delta}_{i} & =\frac{1}{2}\left[b_{i, n}\left(t^{*} ; \lambda_{1}\right) \boldsymbol{\alpha}\right], \quad i=l, l+1, \ldots, m \\
\mathbf{S}_{2} & =\mathbf{S}_{1}+\sum_{i=l}^{m} \boldsymbol{\delta}_{i} b_{i, n}\left(t^{*} ; \lambda_{1}\right) .
\end{aligned}
$$

Due to $t^{*} \in(0,1)$, we know $b_{i, n}\left(t^{*} ; \lambda_{1}\right)>0(i=l, l+1, \ldots, m)$. Substituting (16) into (17), we can get the following equation:

$$
\left[\sum_{i=l}^{m} b_{i, n}^{2}\left(t^{*} ; \lambda_{1}\right)\right] \boldsymbol{\alpha}=2\left(\mathbf{S}_{2}-\mathbf{S}_{1}\right) \text {. }
$$

Namely,

$$
\boldsymbol{\alpha}=\frac{2\left(\mathbf{S}_{2}-\mathbf{S}_{1}\right)}{\sum_{i=l}^{m} b_{i, n}^{2}\left(t^{*} ; \lambda_{1}\right)} .
$$


Substituting (19) into (16) yields

$$
\boldsymbol{\delta}_{i}=\frac{b_{i, n}\left(t^{*} ; \lambda_{1}\right)}{\sum_{i=l}^{m} b_{i, n}^{2}\left(t^{*} ; \lambda_{1}\right)}\left(\mathbf{S}_{2}-\mathbf{S}_{1}\right), \quad i=l, l+1, \ldots, m .
$$

The control points can be modified by (20) such that $\mathbf{p}_{2}\left(t^{*} ; \lambda_{1}\right)=\mathbf{S}_{2}$ is satisfied.

3.2. Tangent Vector Constraint. Let tangent vector of $\lambda$-Bézier curves (10) be $\mathbf{p}_{1}^{\prime}\left(t^{*} ; \lambda_{1}\right)=\mathbf{T}_{1}$ and that of modified curves (11) satisfies $\mathbf{p}_{2}^{\prime}\left(t^{*} ; \lambda_{1}\right)=\mathbf{T}_{2}$. Obviously, the shape of the curves can be preserved well by tangent vector $\mathbf{T}_{2}$, where $\mathbf{T}_{2}$ is dependent on control point $\mathbf{p}_{1}\left(t^{*} ; \lambda_{1}\right)$ and the requirements.

For $n \geq 3$ and $1 \leq l<m \leq n-1$, in order to fix the end-points of modified curve, let $\boldsymbol{\delta}_{i}=\left(\delta_{i}^{x}, \delta_{i}^{y}, \delta_{i}^{z}\right)^{T}$ be the perturbation, and then $\delta_{i}^{z}=0$ if it is plane curve.

According to (11), the following derivative is obtained:

$$
\begin{aligned}
\mathbf{p}_{2}^{\prime}\left(t^{*} ; \lambda_{1}\right)= & \sum_{i=0}^{l-1} \mathbf{P}_{i} b_{i, n}^{\prime}\left(t ; \lambda_{1}\right)+\sum_{i=l}^{m}\left(\mathbf{P}_{i}+\boldsymbol{\delta}_{i}\right) b_{i, n}^{\prime}\left(t ; \lambda_{1}\right) \\
& +\sum_{i=m+1}^{n} \mathbf{P}_{i} b_{i, n}^{\prime}\left(t ; \lambda_{1}\right) \\
= & \sum_{i=0}^{n} \mathbf{P}_{i} b_{i, n}^{\prime}\left(t ; \lambda_{1}\right)+\sum_{i=l}^{m} \boldsymbol{\delta}_{i} b_{i, n}^{\prime}\left(t ; \lambda_{1}\right) \\
= & \mathbf{T}_{1}+\sum_{i=l}^{m} \boldsymbol{\delta}_{i} b_{i, n}^{\prime}\left(t ; \lambda_{1}\right)=\mathbf{T}_{2} .
\end{aligned}
$$

Notice that $\boldsymbol{\delta}_{i}$ is nonunique if $m>l$, and it can be computed by constrained optimization with $\sum_{i=l}^{m}\left\|\boldsymbol{\delta}_{i}\right\|^{2}$ being minimum. The Lagrange function is defined as follows:

$$
L=\sum_{i=l}^{m}\left\|\boldsymbol{\delta}_{i}\right\|^{2}+\boldsymbol{\beta}^{T}\left[\mathbf{T}_{2}-\mathbf{p}_{2}^{\prime}\left(t^{*} ; \lambda_{1}\right)\right]
$$

where $\beta^{T}=\left(\beta^{x}, \beta^{y}, \beta^{z}\right)$ is the Lagrange multiplier and $\|\cdot\|$ is Euclidean norm.

Theorem 5. When $t^{*} \in(0,1),(22)$ has a unique solution if $m>l$ or $m=l$ and $t^{*}$ is not the maximum point.

Proof. Letting

$$
\begin{aligned}
& \frac{\partial L}{\partial \delta_{i}^{x}}=\frac{\partial L}{\partial \delta_{i}^{y}}=\frac{\partial L}{\partial \delta_{i}^{z}}=0, \quad i=l, l+1, \ldots, m \\
& \frac{\partial L}{\partial \beta^{x}}=\frac{\partial L}{\partial \beta^{y}}=\frac{\partial L}{\partial \beta^{z}}=0,
\end{aligned}
$$

we can obtain

$$
\begin{aligned}
& \boldsymbol{\delta}_{i}=\frac{1}{2}\left[b_{i, n}^{\prime}\left(t^{*} ; \lambda_{1}\right) \boldsymbol{\beta}\right], \quad i=l, l+1, \ldots, m \\
& \mathbf{T}_{2}=\mathbf{T}_{1}+\sum_{i=l}^{m} \boldsymbol{\delta}_{i} b_{i, n}^{\prime}\left(t^{*} ; \lambda_{1}\right) .
\end{aligned}
$$

When $m>l, b_{i, n}^{\prime}\left(t^{*} ; \lambda\right)(i=l, l+1, \ldots, m)$ are not all zero. When $m>l$ or $m=l$ and $t^{*}$ is not the maximum point, we have $b_{i, n}^{\prime}\left(t^{*} ; \lambda\right) \neq 0$. Substituting (25) into (24), we can get the following expression:

$$
\boldsymbol{\beta}=\frac{2\left(\mathbf{T}_{2}-\mathbf{T}_{1}\right)}{\sum_{i=l}^{m}\left[b_{i, n}^{\prime}\left(t^{*} ; \lambda_{1}\right)\right]^{2}}
$$

Combining (24) and (26), we have

$$
\begin{aligned}
& \boldsymbol{\delta}_{i}=\frac{b_{i, n}^{\prime}\left(t^{*} ; \lambda_{1}\right)}{\sum_{i=l}^{m}\left[b_{i, n}^{\prime}\left(t^{*} ; \lambda_{1}\right)\right]^{2}}\left(\mathbf{T}_{2}-\mathbf{T}_{1}\right) \\
& i=l, l+1, \ldots, m \text {. }
\end{aligned}
$$

The control points can be modified according to (27) such that $\mathbf{p}_{2}^{\prime}\left(t^{*} ; \lambda_{1}\right)=\mathbf{T}_{2}$ is satisfied.

3.3. Position Vector and Tangent Vector Constraints. Let position vector of $\lambda$-Bézier curves $(10)$ be $\mathbf{p}_{1}\left(t^{*} ; \lambda_{1}\right)=\mathbf{S}_{1}$ and let tangent vector be $\mathbf{p}_{1}^{\prime}\left(t^{*} ; \lambda_{1}\right)=\mathbf{T}_{1}$; then, those of modified curves (11) satisfy $\mathbf{p}_{2}\left(t^{*} ; \lambda_{1}\right)=\mathbf{S}_{2}$ and $\mathbf{p}_{2}^{\prime}\left(t^{*} ; \lambda_{1}\right)=\mathbf{T}_{2}$, respectively, and here

$$
\begin{aligned}
& \mathbf{p}_{2}\left(t^{*} ; \lambda_{1}\right)=\mathbf{S}_{1}+\sum_{i=l}^{m} \boldsymbol{\delta}_{i} b_{i, n}\left(t^{*} ; \lambda_{1}\right)=\mathbf{S}_{2}, \\
& \mathbf{p}_{2}^{\prime}\left(t^{*} ; \lambda_{1}\right)=\mathbf{T}_{1}+\sum_{i=l}^{m} \boldsymbol{\delta}_{i} b_{i, n}^{\prime}\left(t^{*} ; \lambda_{1}\right)=\mathbf{T}_{2} .
\end{aligned}
$$

When $n \geq 3$ and $1 \leq l<m \leq n-1$, (11) has at least two perturbations, which can be computed by constrained optimization with $\sum_{i=l}^{m}\left\|\boldsymbol{\delta}_{i}\right\|^{2}$ being minimum. The Lagrange function is defined as follows:

$$
\begin{aligned}
L= & \sum_{i=l}^{m}\left\|\boldsymbol{\delta}_{i}\right\|^{2}+\boldsymbol{\alpha}^{T}\left[\mathbf{S}_{2}-\mathbf{p}_{2}\left(t^{*} ; \lambda_{1}\right)\right] \\
& +\boldsymbol{\beta}^{T}\left[\mathbf{T}_{2}-\mathbf{p}_{2}^{\prime}\left(t^{*} ; \lambda_{1}\right)\right]
\end{aligned}
$$

where $\boldsymbol{\alpha}^{T}=\left(\alpha^{x}, \alpha^{y}, \alpha^{z}\right)$ and $\beta^{T}=\left(\beta^{x}, \beta^{y}, \beta^{z}\right)$ are the Lagrange multipliers and $\|\cdot\|$ is Euclidean norm.

Theorem 6. When $t^{*} \in(0,1)$, (29) has a unique solution for $n \geq 3$ and $1 \leq l<m \leq n-1$.

Proof. Setting

$$
\begin{aligned}
\frac{\partial L}{\partial \delta_{i}^{x}} & =\frac{\partial L}{\partial \delta_{i}^{y}}=\frac{\partial L}{\partial \delta_{i}^{z}}=0, \quad i=l, l+1, \ldots, m \\
\frac{\partial L}{\partial \alpha^{x}} & =\frac{\partial L}{\partial \alpha^{y}}=\frac{\partial L}{\partial \alpha^{z}}=0, \\
\frac{\partial L}{\partial \beta^{x}} & =\frac{\partial L}{\partial \beta^{y}}=\frac{\partial L}{\partial \beta^{z}}=0,
\end{aligned}
$$


we can obtain

$$
\begin{aligned}
& \boldsymbol{\delta}_{i}=\frac{1}{2}\left[b_{i, n}\left(t^{*} ; \lambda_{1}\right) \boldsymbol{\alpha}+b_{i, n}^{\prime}\left(t^{*} ; \lambda_{1}\right) \boldsymbol{\beta}\right], \\
& \quad i=l, l+1, \ldots, m \\
& \mathbf{S}_{2}=\mathbf{S}_{1}+\sum_{i=l}^{m} \boldsymbol{\delta}_{i} b_{i, n}\left(t^{*} ; \lambda_{1}\right) \\
& \mathbf{T}_{2}=\mathbf{T}_{1}+\sum_{i=l}^{m} \boldsymbol{\delta}_{i} b_{i, n}^{\prime}\left(t^{*} ; \lambda_{1}\right) .
\end{aligned}
$$

Substituting (31) into (32) and (33), we have the following equations:

$$
\begin{aligned}
& {\left[\sum_{i=l}^{m} b_{i, n}^{2}\left(t^{*} ; \lambda_{1}\right)\right] \boldsymbol{\alpha}+\left[\sum_{i=l}^{m} b_{i, n}\left(t^{*} ; \lambda_{1}\right) b_{i, n}^{\prime}\left(t^{*} ; \lambda_{1}\right)\right] \boldsymbol{\beta}} \\
& \quad=2\left(\mathbf{S}_{2}-\mathbf{S}_{1}\right), \\
& {\left[\sum_{i=l}^{m} b_{i, n}\left(t^{*} ; \lambda_{1}\right) b_{i, n}^{\prime}\left(t^{*} ; \lambda_{1}\right)\right] \boldsymbol{\alpha}+\sum_{i=l}^{m}\left[b_{i, n}^{\prime}\left(t^{*} ; \lambda_{1}\right)\right]^{2} \boldsymbol{\beta}} \\
& =2\left(\mathbf{T}_{2}-\mathbf{T}_{1}\right) .
\end{aligned}
$$

Denote

$$
\begin{gathered}
\Delta=\left[\sum_{i=l}^{m} b_{i, n}^{2}\left(t^{*} ; \lambda_{1}\right)\right]\left[\sum_{i=l}^{m}\left[b_{i, n}^{\prime}\left(t^{*} ; \lambda_{1}\right)\right]^{2}\right] \\
-\left[\sum_{i=l}^{m} b_{i, n}\left(t^{*} ; \lambda_{1}\right) b_{i, n}^{\prime}\left(t^{*} ; \lambda_{1}\right)\right]^{2} .
\end{gathered}
$$

We know $\Delta \geq 0$ by inequality of Cauchy-Schwarz. Further, we have

$$
\begin{aligned}
\Delta & =\sum_{l \leq i<j \leq m}\left[b_{i, n}\left(t^{*} ; \lambda_{1}\right) b_{j, n}^{\prime}\left(t^{*} ; \lambda_{1}\right)\right. \\
& \left.-b_{i, n}^{\prime}\left(t^{*} ; \lambda_{1}\right) b_{j, n}\left(t^{*} ; \lambda_{1}\right)\right]^{2} .
\end{aligned}
$$

For $1 \leq l<m \leq n-1$ and $t^{*} \in(0,1)$, the following inequality can be easily obtained

$$
\begin{aligned}
& b_{i, n}\left(t^{*} ; \lambda_{1}\right) b_{i+1, n}^{\prime}\left(t^{*} ; \lambda_{1}\right)-b_{i, n}^{\prime}\left(t^{*} ; \lambda_{1}\right) b_{i+1, n}\left(t^{*} ; \lambda_{1}\right) \\
& \quad>0,
\end{aligned}
$$

which implies $\Delta>0$. The following equations are presented by (34):

$$
\begin{aligned}
\boldsymbol{\alpha} & =\frac{2}{\Delta}\left[\left(\sum_{i=l}^{m}\left[b_{i, n}^{\prime}\left(t^{*} ; \lambda_{1}\right)\right]^{2}\right)\left(\mathbf{S}_{2}-\mathbf{S}_{1}\right)\right. \\
& \left.-\left(\sum_{i=l}^{m} b_{i, n}\left(t^{*} ; \lambda_{1}\right) b_{i, n}^{\prime}\left(t^{*} ; \lambda_{1}\right)\right)\left(\mathbf{T}_{2}-\mathbf{T}_{1}\right)\right], \\
\boldsymbol{\beta} & =\frac{2}{\Delta}\left[\left(\sum_{i=l}^{m} b_{i, n}^{2}\left(t^{*} ; \lambda_{1}\right)\right)\left(\mathbf{T}_{2}-\mathbf{T}_{1}\right)\right. \\
& \left.-\left(\sum_{i=l}^{m} b_{i, n}\left(t^{*} ; \lambda_{1}\right) b_{i, n}^{\prime}\left(t^{*} ; \lambda_{1}\right)\right)\left(\mathbf{S}_{2}-\mathbf{S}_{1}\right)\right] .
\end{aligned}
$$

Accordingly, when $i=l, l+1, \ldots, m$, substituting (38) into (31) leads to

$$
\begin{aligned}
\boldsymbol{\delta}_{i} & =\frac{\mathbf{S}_{2}-\mathbf{S}_{1}}{\Delta}\left[\left(\sum_{i=l}^{m}\left[b_{i, n}^{\prime}\left(t^{*} ; \lambda_{1}\right)\right]^{2}\right) b_{i, n}\left(t^{*} ; \lambda_{1}\right)\right. \\
& \left.-\left(\sum_{i=l}^{m} b_{i, n}\left(t^{*} ; \lambda_{1}\right) b_{i, n}^{\prime}\left(t^{*} ; \lambda_{1}\right)\right) b_{i, n}^{\prime}\left(t^{*} ; \lambda_{1}\right)\right] \\
& +\frac{\mathbf{T}_{2}-\mathbf{T}_{1}}{\Delta}\left[\left(\sum_{i=l}^{m} b_{i, n}^{2}\left(t^{*} ; \lambda_{1}\right)\right) b_{i, n}^{\prime}\left(t^{*} ; \lambda_{1}\right)\right. \\
& \left.-\left(\sum_{i=l}^{m} b_{i, n}\left(t^{*} ; \lambda_{1}\right) b_{i, n}^{\prime}\left(t^{*} ; \lambda_{1}\right)\right) b_{i, n}\left(t^{*} ; \lambda_{1}\right)\right] .
\end{aligned}
$$

The control points can be modified by (39) such that $\mathbf{p}_{2}^{\prime}\left(t^{*} ; \lambda_{1}\right)=\mathbf{T}_{2}$ and $\mathbf{p}_{2}^{\prime}\left(t^{*} ; \lambda_{1}\right)=\mathbf{T}_{2}$.

3.4. Position Vector, Tangent Vector, and Shape Parameter Constraints. Let position vector of $\lambda$-Bézier curves (10) be $\mathbf{p}_{1}\left(t^{*} ; \lambda_{1}\right)=\mathbf{S}_{1}$ and let tangent vector be $\mathbf{p}_{1}^{\prime}\left(t^{*} ; \lambda_{1}\right)=\mathbf{T}_{1}$; then position vector and tangent vector of modified curves (12) satisfy $\mathbf{p}_{3}\left(t^{*} ; \lambda_{2}\right)=\mathbf{S}_{2}$ and $\mathbf{p}_{3}^{\prime}\left(t^{*} ; \lambda_{2}\right)=\mathbf{T}_{2}$, respectively, and here

$$
\begin{aligned}
\mathbf{p}_{3}\left(t^{*} ; \lambda_{2}\right)= & \mathbf{S}_{1}+\sum_{i=0}^{l-1} \mathbf{P}_{i}\left[b_{i, n}\left(t ; \lambda_{2}\right)-b_{i, n}\left(t ; \lambda_{1}\right)\right] \\
& +\sum_{i=l}^{m} \mathbf{P}_{i}\left[b_{i, n}\left(t ; \lambda_{2}\right)-b_{i, n}\left(t ; \lambda_{1}\right)\right] \\
& +\sum_{i=l}^{m} \boldsymbol{\delta}_{i} b_{i, n}\left(t ; \lambda_{2}\right) \\
& +\sum_{i=m+1}^{n} \mathbf{P}_{i}\left[b_{i, n}\left(t ; \lambda_{2}\right)-b_{i, n}\left(t ; \lambda_{1}\right)\right] \\
= & \mathbf{S}_{2},
\end{aligned}
$$




$$
\begin{array}{rlr}
\mathbf{p}_{3}^{\prime}\left(t^{*} ; \lambda_{2}\right)= & \mathbf{T}_{1}+\sum_{i=0}^{l-1} \mathbf{P}_{i}\left[b_{i, n}^{\prime}\left(t ; \lambda_{2}\right)-b_{i, n}^{\prime}\left(t ; \lambda_{1}\right)\right] & \mathbf{T}_{2}=\mathbf{T}_{1}+\sum_{i=0}^{l-1} \mathbf{P}_{i}\left[b_{i, n}^{\prime}\left(t ; \lambda_{2}\right)-b_{i, n}^{\prime}\left(t ; \lambda_{1}\right)\right] \\
& +\sum_{i=l}^{m} \mathbf{P}_{i}\left[b_{i, n}^{\prime}\left(t ; \lambda_{2}\right)-b_{i, n}^{\prime}\left(t ; \lambda_{1}\right)\right] & +\sum_{i=l}^{m} \mathbf{P}_{i}\left[b_{i, n}^{\prime}\left(t ; \lambda_{2}\right)-b_{i, n}^{\prime}\left(t ; \lambda_{1}\right)\right] \\
& +\sum_{i=l}^{m} \boldsymbol{\delta}_{i} b_{i, n}^{\prime}\left(t ; \lambda_{2}\right) & +\sum_{i=l}^{m} \boldsymbol{\delta}_{i} b_{i, n}^{\prime}\left(t ; \lambda_{2}\right) \\
& +\sum_{i=m+1}^{n} \mathbf{P}_{i}\left[b_{i, n}^{\prime}\left(t ; \lambda_{2}\right)-b_{i, n}^{\prime}\left(t ; \lambda_{1}\right)\right] & +\sum_{i=m+1}^{n} \mathbf{P}_{i}\left[b_{i, n}^{\prime}\left(t ; \lambda_{2}\right)-b_{i, n}^{\prime}\left(t ; \lambda_{1}\right)\right] .
\end{array}
$$

Substituting (43) into (44) and (45), it follows that

When $n \geq 3$ and $1 \leq l<m \leq n-1$, then (12) has at least two perturbations which can be computed by constrained optimization with $\sum_{i=l}^{m}\left\|\boldsymbol{\delta}_{i}\right\|^{2}$ being minimum. The Lagrange function is defined as follows:

$$
\begin{aligned}
L= & \sum_{i=l}^{m}\left\|\boldsymbol{\delta}_{i}\right\|^{2}+\boldsymbol{\alpha}^{T}\left[\mathbf{S}_{2}-\mathbf{p}_{3}\left(t^{*} ; \lambda_{2}\right)\right] \\
& +\boldsymbol{\beta}^{T}\left[\mathbf{T}_{2}-\mathbf{p}_{3}^{\prime}\left(t^{*} ; \lambda_{2}\right)\right],
\end{aligned}
$$

where $\boldsymbol{\alpha}^{T}=\left(\alpha^{x}, \alpha^{y}, \alpha^{z}\right)$ and $\beta^{T}=\left(\beta^{x}, \beta^{y}, \beta^{z}\right)$ are the Lagrange multipliers and $\|\cdot\|$ is Euclidean norm.

Theorem 7. When $t^{*} \in(0,1)$, (41) has a unique solution for $n \geq 3$ and $1 \leq l<m \leq n-1$.

Proof. Letting

$$
\begin{aligned}
\frac{\partial L}{\partial \delta_{i}^{x}} & =\frac{\partial L}{\partial \delta_{i}^{y}}=\frac{\partial L}{\partial \delta_{i}^{z}}=0, \quad i=l, l+1, \ldots, m \\
\frac{\partial L}{\partial \alpha^{x}} & =\frac{\partial L}{\partial \alpha^{y}}=\frac{\partial L}{\partial \alpha^{z}}=0, \\
\frac{\partial L}{\partial \beta^{x}} & =\frac{\partial L}{\partial \beta^{y}}=\frac{\partial L}{\partial \beta^{z}}=0,
\end{aligned}
$$

we can obtain

$$
\begin{aligned}
\boldsymbol{\delta}_{i}=\frac{1}{2}\left[b_{i, n}\left(t^{*} ; \lambda_{2}\right) \boldsymbol{\alpha}+b_{i, n}^{\prime}\left(t^{*} ; \lambda_{2}\right) \boldsymbol{\beta}\right] & i=l, l+1, \ldots, m \\
\mathbf{S}_{2}= & \mathbf{S}_{1}+\sum_{i=0}^{l-1} \mathbf{P}_{i}\left[b_{i, n}\left(t ; \lambda_{2}\right)-b_{i, n}\left(t ; \lambda_{1}\right)\right] \\
& +\sum_{i=l}^{m} \mathbf{P}_{i}\left[b_{i, n}\left(t ; \lambda_{2}\right)-b_{i, n}\left(t ; \lambda_{1}\right)\right] \\
& +\sum_{i=l}^{m} \boldsymbol{\delta}_{i} b_{i, n}\left(t ; \lambda_{2}\right) \\
& +\sum_{i=m+1}^{n} \mathbf{P}_{i}\left[b_{i, n}\left(t ; \lambda_{2}\right)-b_{i, n}\left(t ; \lambda_{1}\right)\right]
\end{aligned}
$$

$$
\begin{aligned}
& {\left[\sum_{i=l}^{m} b_{i, n}\left(t^{*} ; \lambda_{2}\right) b_{i, n}^{\prime}\left(t^{*} ; \lambda_{2}\right)\right] \boldsymbol{\alpha}+\sum_{i=l}^{m}\left[b_{i, n}^{\prime}\left(t^{*} ; \lambda_{2}\right)\right]^{2} \boldsymbol{\beta}} \\
& \quad=2\left(\mathbf{T}_{2}-\sum_{i=0}^{n} \mathbf{P}_{i} b_{i, n}^{\prime}\left(t ; \lambda_{2}\right)\right) .
\end{aligned}
$$

Let

$$
\begin{aligned}
\Delta= & {\left[\sum_{i=l}^{m} b_{i, n}^{2}\left(t^{*} ; \lambda_{2}\right)\right]\left[\sum_{i=l}^{m}\left[b_{i, n}^{\prime}\left(t^{*} ; \lambda_{2}\right)\right]^{2}\right] } \\
& -\left[\sum_{i=l}^{m} b_{i, n}\left(t^{*} ; \lambda_{2}\right) b_{i, n}^{\prime}\left(t^{*} ; \lambda_{2}\right)\right]^{2} .
\end{aligned}
$$

We know $\Delta \geq 0$ by inequality of Cauchy-Schwarz. Moreover, we have

$$
\begin{aligned}
\Delta & =\sum_{l \leq i<j \leq m}\left[b_{i, n}\left(t^{*} ; \lambda_{2}\right) b_{j, n}^{\prime}\left(t^{*} ; \lambda_{2}\right)\right. \\
& \left.-b_{i, n}^{\prime}\left(t^{*} ; \lambda_{2}\right) b_{j, n}\left(t^{*} ; \lambda_{2}\right)\right]^{2}
\end{aligned}
$$

For $1 \leq l<m \leq n-1$ and $t^{*} \in(0,1)$, the following inequality can be easily obtained

$$
\begin{aligned}
& b_{i, n}\left(t^{*} ; \lambda_{2}\right) b_{i+1, n}^{\prime}\left(t^{*} ; \lambda_{2}\right)-b_{i, n}^{\prime}\left(t^{*} ; \lambda_{2}\right) b_{i+1, n}\left(t^{*} ; \lambda_{2}\right) \\
& \quad>0
\end{aligned}
$$




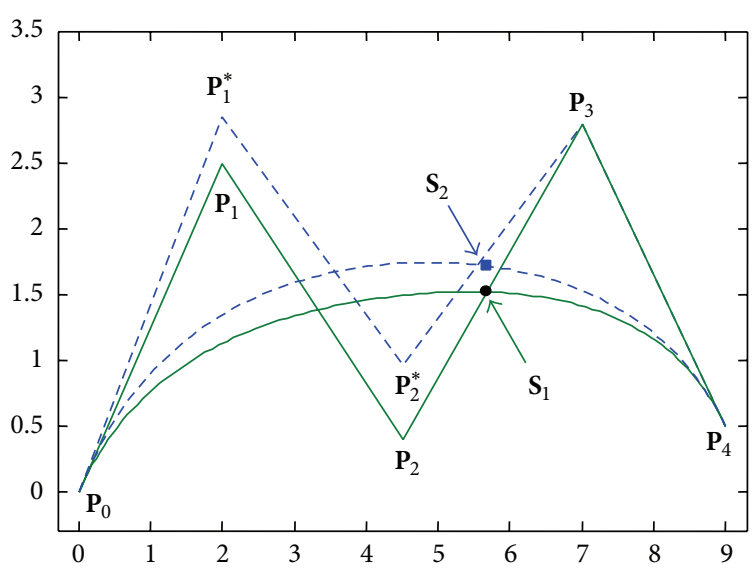

(a) Modification of control points $\mathbf{P}_{1}, \mathbf{P}_{2}$

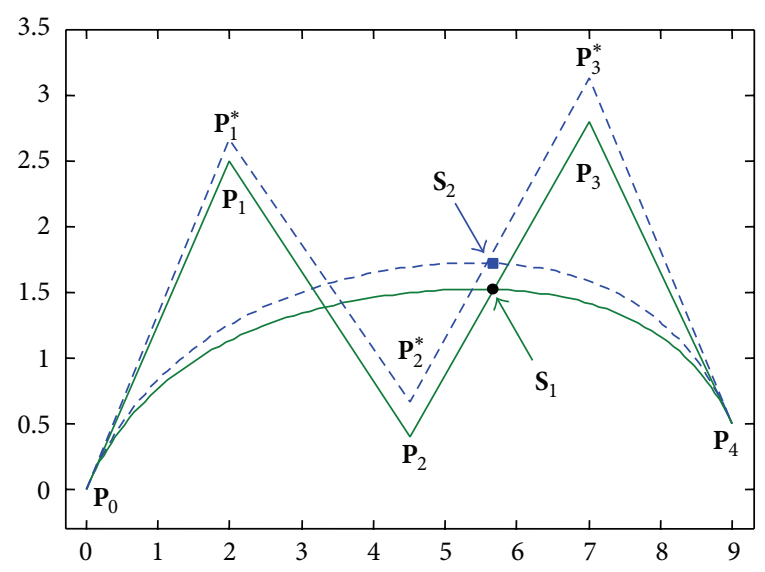

(b) Modification of control points $\mathbf{P}_{1}, \mathbf{P}_{2}$, and $\mathbf{P}_{3}$

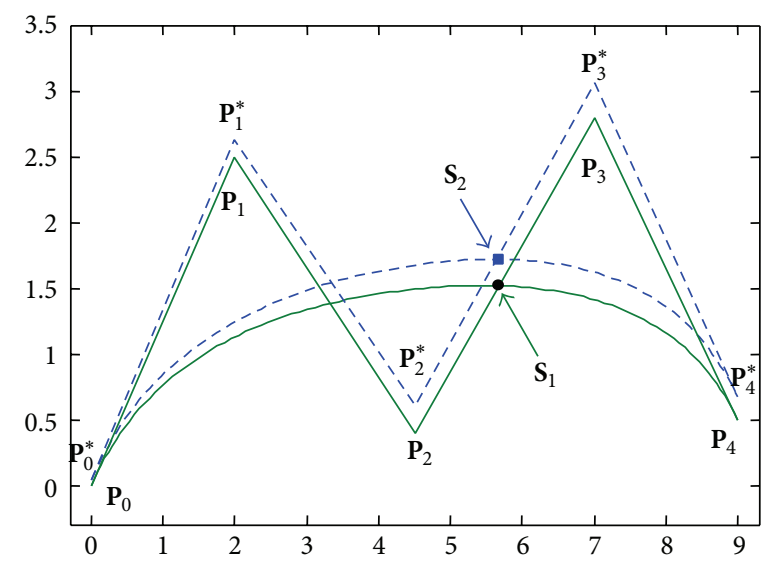

(c) Modification of control points $\mathbf{P}_{0}, \mathbf{P}_{1}, \mathbf{P}_{2}, \mathbf{P}_{3}$, and $\mathbf{P}_{4}$

FIGURE 2: Shape modification for quartic $\lambda$-Bézier curve based on position vector constraint of single target point.

which implies $\Delta>0$. The following equations are deduced by (46):

$$
\begin{aligned}
\boldsymbol{\alpha} & =\frac{2}{\Delta}\left[\left(\sum_{i=l}^{m}\left[b_{i, n}^{\prime}\left(t^{*} ; \lambda_{2}\right)\right]^{2}\right)\left(\mathbf{S}_{2}-\sum_{i=0}^{n} \mathbf{P}_{i} b_{i, n}\left(t ; \lambda_{2}\right)\right)\right. \\
& -\left(\sum_{i=l}^{m} b_{i, n}\left(t^{*} ; \lambda_{2}\right) b_{i, n}^{\prime}\left(t^{*} ; \lambda_{2}\right)\right) \\
& \left.\cdot\left(\mathbf{T}_{2}-\sum_{i=0}^{n} \mathbf{P}_{i} b_{i, n}^{\prime}\left(t ; \lambda_{2}\right)\right)\right], \\
\boldsymbol{\beta}= & \frac{2}{\Delta}\left[\left(\sum_{i=l}^{m} b_{i, n}^{2}\left(t^{*} ; \lambda_{2}\right)\right)\left(\mathbf{T}_{2}-\sum_{i=0}^{n} \mathbf{P}_{i} b_{i, n}^{\prime}\left(t ; \lambda_{2}\right)\right)\right. \\
& -\left(\sum_{i=l}^{m} b_{i, n}\left(t^{*} ; \lambda_{2}\right) b_{i, n}^{\prime}\left(t^{*} ; \lambda_{2}\right)\right) \\
& \left.\cdot\left(\mathbf{S}_{2}-\sum_{i=0}^{n} \mathbf{P}_{i} b_{i, n}\left(t ; \lambda_{2}\right)\right)\right] .
\end{aligned}
$$

Accordingly, for $i=l, l+1, \ldots m$, substituting (50) into (43), we have

$$
\begin{aligned}
\boldsymbol{\delta}_{i} & =\frac{\mathbf{S}_{2}-\sum_{i=0}^{n} \mathbf{P}_{i} b_{i, n}\left(t ; \lambda_{2}\right)}{\Delta}\left[\left(\sum_{i=l}^{m}\left[b_{i, n}^{\prime}\left(t^{*} ; \lambda_{2}\right)\right]^{2}\right)\right. \\
& \cdot b_{i, n}\left(t^{*} ; \lambda_{2}\right)-\left(\sum_{i=l}^{m} b_{i, n}\left(t^{*} ; \lambda_{2}\right) b_{i, n}^{\prime}\left(t^{*} ; \lambda_{2}\right)\right) \\
& \left.\cdot b_{i, n}^{\prime}\left(t^{*} ; \lambda_{2}\right)\right] \\
& +\frac{\mathbf{T}_{2}-\sum_{i=0}^{n} \mathbf{P}_{i} b_{i, n}^{\prime}\left(t ; \lambda_{2}\right)}{\Delta}\left[\left(\sum_{i=l}^{m} b_{i, n}^{2}\left(t^{*} ; \lambda_{2}\right)\right)\right. \\
& \cdot b_{i, n}^{\prime}\left(t^{*} ; \lambda_{2}\right)-\left(\sum_{i=l}^{m} b_{i, n}\left(t^{*} ; \lambda_{2}\right) b_{i, n}^{\prime}\left(t^{*} ; \lambda_{2}\right)\right) \\
& \left.\cdot b_{i, n}\left(t^{*} ; \lambda_{2}\right)\right] .
\end{aligned}
$$




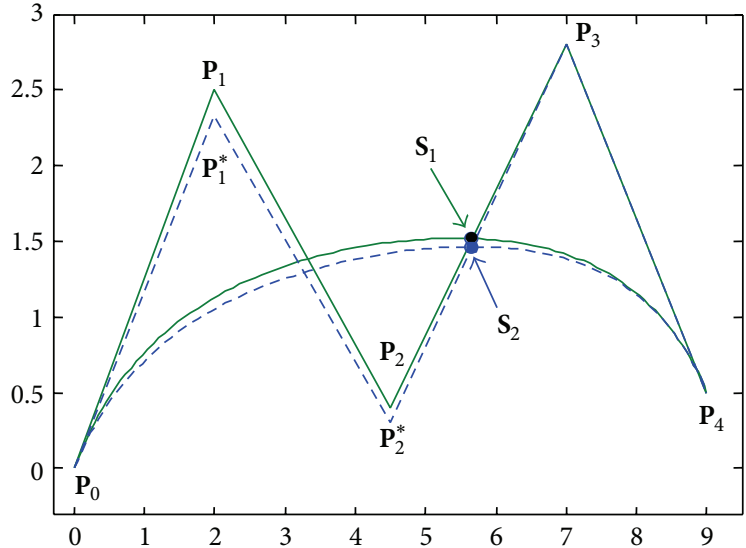

(a) Modification of control points $\mathbf{P}_{1}, \mathbf{P}_{2}$

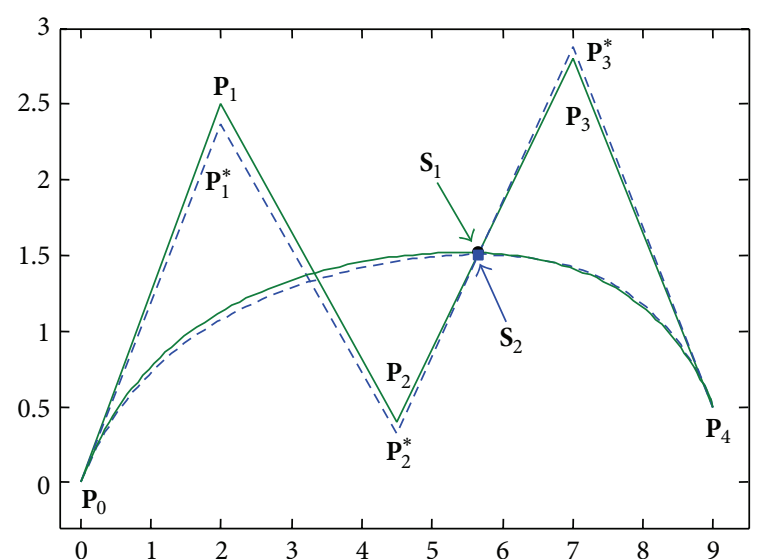

(b) Modification of control points $\mathbf{P}_{1}, \mathbf{P}_{2}$, and $\mathbf{P}_{3}$

FIgURE 3: Shape modification for quartic $\lambda$-Bézier curve based on tangent vector constraint of single target point.

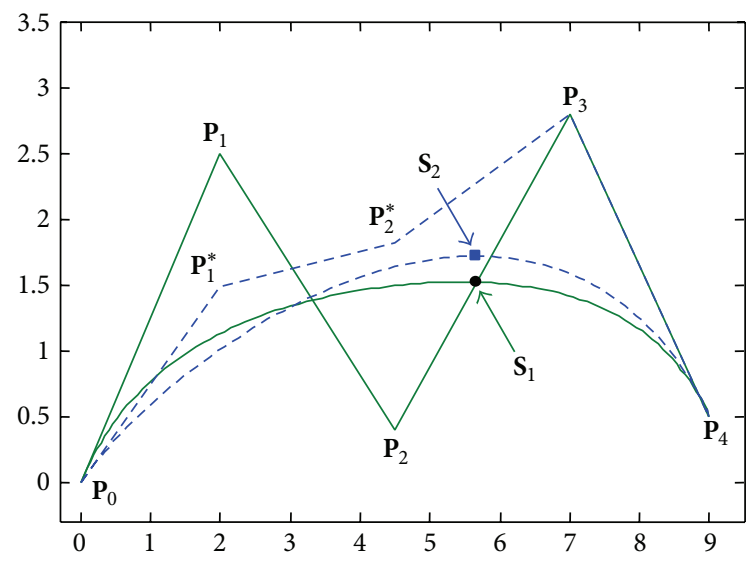

(a) Modification of control points $\mathbf{P}_{1}, \mathbf{P}_{2}$

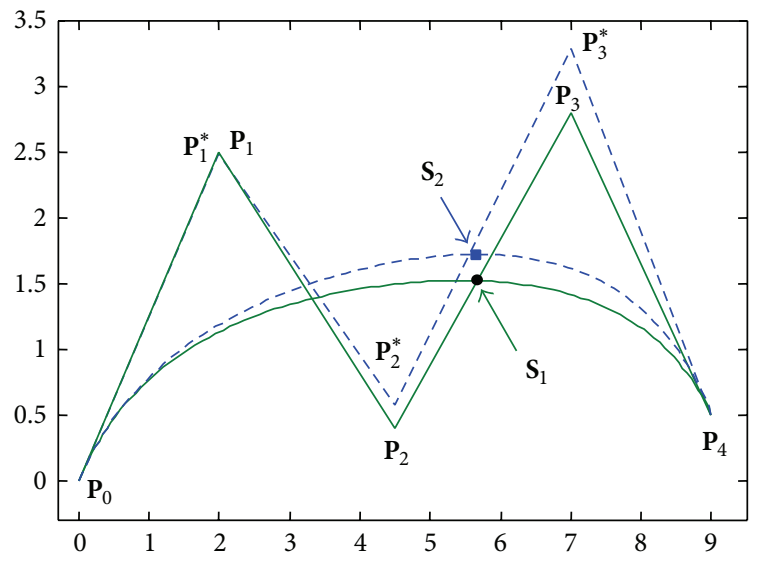

(b) Modification of control points $\mathbf{P}_{1}, \mathbf{P}_{2}$, and $\mathbf{P}_{3}$

FIGURE 4: Shape modification for quartic $\lambda$-Bézier curve based on position vector and tangent vector constraint of single target point.

The control points can be modified by (51) such that $\mathbf{p}_{3}\left(t^{*} ; \lambda_{2}\right)=\mathbf{S}_{2}$ and $\mathbf{p}_{3}^{\prime}\left(t^{*} ; \lambda_{2}\right)=\mathbf{T}_{2}$.

\section{Practical Examples}

In this section, we will give three examples to show the effects of the proposed method.

Example 1. Given shape parameter $\lambda_{1}=-1$ and control points $\left\{\mathbf{P}_{0}=(0,0), \mathbf{P}_{1}=(2,2.5), \mathbf{P}_{2}=(4.5,0.4), \mathbf{P}_{3}=\right.$ $\left.(7,2.8), \mathbf{P}_{4}=(9,0.5)\right\}$, we can construct an original quartic $\lambda$-Bézier curve. In Figures 2, 3, and 4, the shape modification of $\lambda$-Bézier curves by constrained optimization of position vector and tangent vector with single target point $\mathbf{S}_{1}=$ $\mathbf{p}_{1}\left(0.6 ; \lambda_{1}\right)$ are obtained, where original curves are shown as green solid lines and modified curves are shown as blue dashed lines. Original target points $\mathbf{S}_{1}$ are black dot and modified target points $\mathbf{S}_{2}=\mathbf{p}_{2}\left(0.6 ; \lambda_{1}\right)$ are blue square point. $\mathbf{P}_{i}^{*}(i=0,1,2,3,4)$ are control points of modified curves.
Figure 5 illustrates the shape modification for quartic $\lambda$ Bézier curves by constrained optimization of position vector, tangent vector, and shape parameter of single target point $\mathbf{S}_{1}=\mathbf{p}_{1}\left(0.6 ; \lambda_{1}\right)$. In Figure 5 , the shape parameter $\lambda_{1}$ of original curves equals -1 , while the shape parameter $\lambda_{2}$ of modified curves equals 1 . The control points $\mathbf{P}_{i}(i=$ $0,1,2,3,4)$ of original curves are the same as those of the curves in Figure $4 . \mathbf{P}_{i}^{*}(i=1,2,3)$ are control points of modified curves. Original curves are shown as green solid lines and modified curves are shown as blue dashed lines, where original target points $\mathbf{S}_{1}$ are black dot and modified target points $\mathbf{S}_{2}=\mathbf{p}_{3}\left(0.6 ; \lambda_{2}\right)$ are blue square point.

Example 2. Given shape parameter $\lambda_{1}=1$ and control points $\left\{\mathbf{P}_{0}=(0,0), \mathbf{P}_{1}=(1,2), \mathbf{P}_{2}=(2,2.3), \mathbf{P}_{3}=(3\right.$, $0.4), \mathbf{P}_{4}=(5,0.8), \mathbf{P}_{5}=(6,2.1), \mathbf{P}_{6}=(8,2.7), \mathbf{P}_{7}=$ $(9,0.3)\}$, we can construct an original $\lambda$-Bézier curve of degree 7. Figure 6 illustrates the shape modification for it by constrained optimization of position and tangent vector of single target point $\mathbf{S}_{1}=\mathbf{p}_{1}\left(0.6 ; \lambda_{1}\right)$, where original curves are 


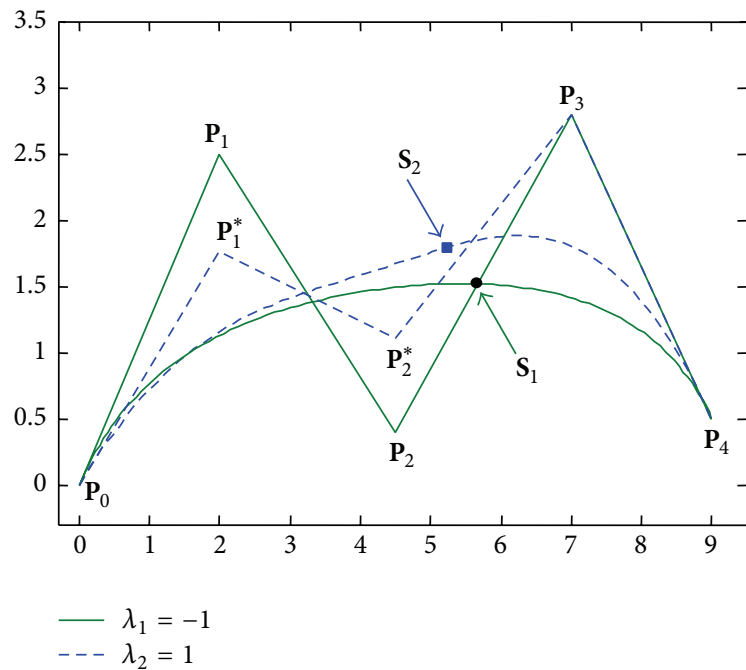

(a) Modification of control points $\mathbf{P}_{1}, \mathbf{P}_{2}$

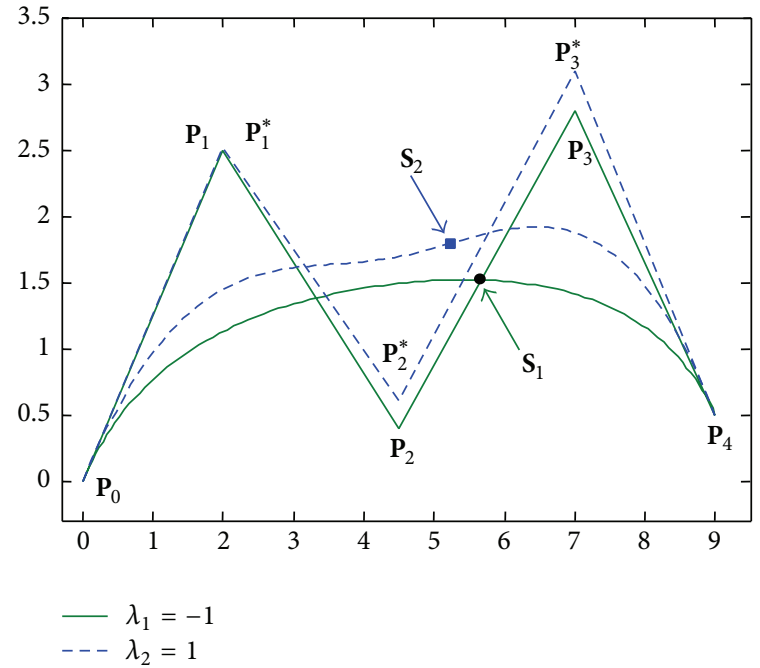

(b) Modification of control points $\mathbf{P}_{1}, \mathbf{P}_{2}$, and $\mathbf{P}_{3}$

FIGURE 5: Shape modification for quartic $\lambda$-Bézier curve based on position vector, tangent vector, and shape parameter constraint of single target point.

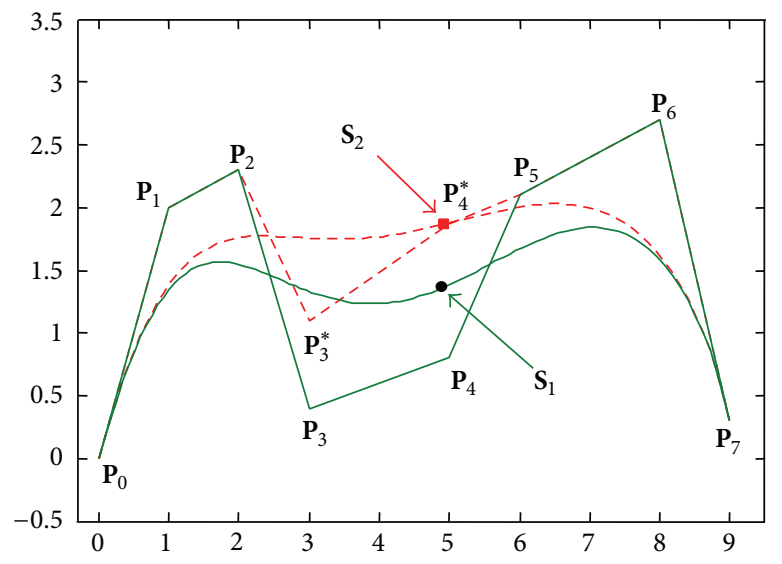

(a) Position vector constraint

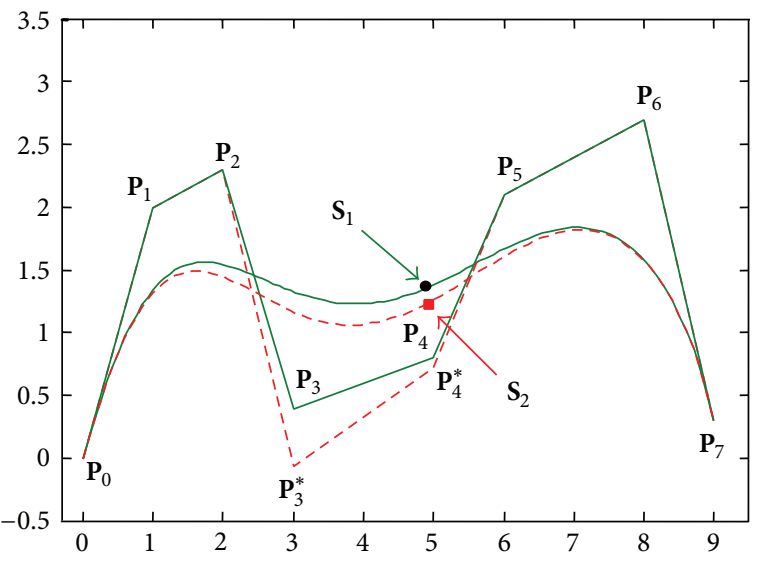

(b) Tangent vector constraint

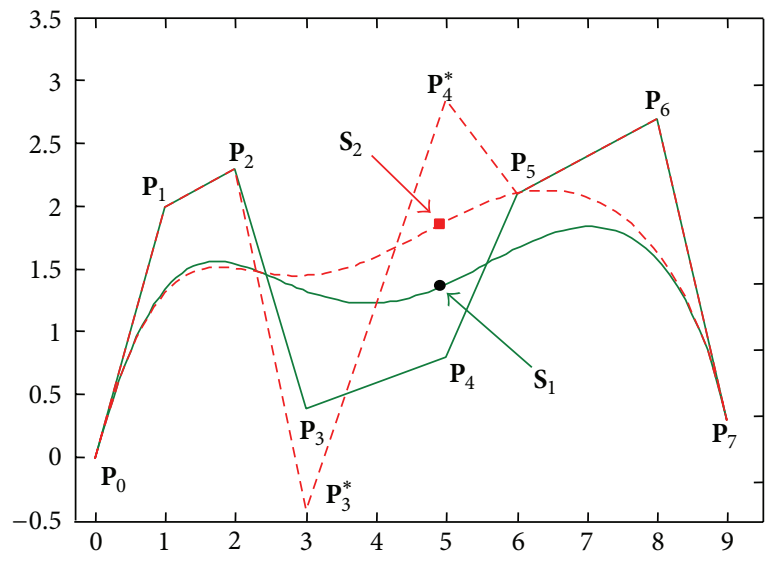

(c) Position vector and tangent vector constraints

FIGURE 6: Shape modification for $\lambda$-Bézier curves of degree seven with single target point. 


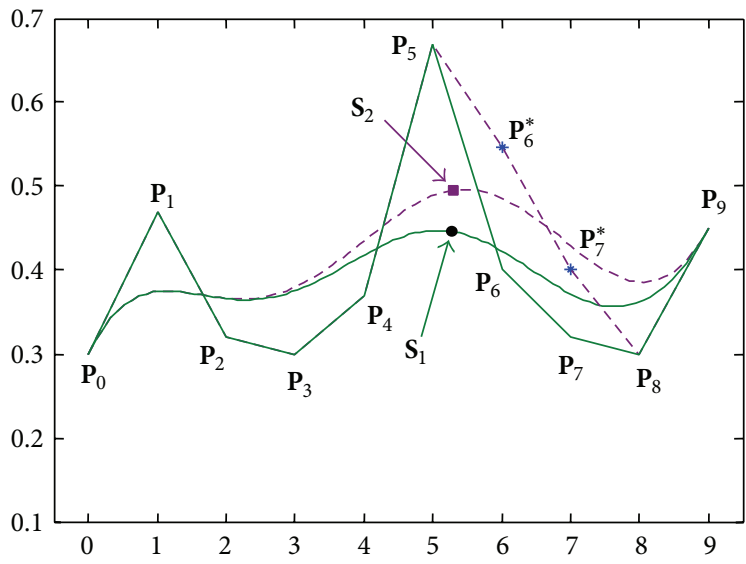

(a) Position vector constraint

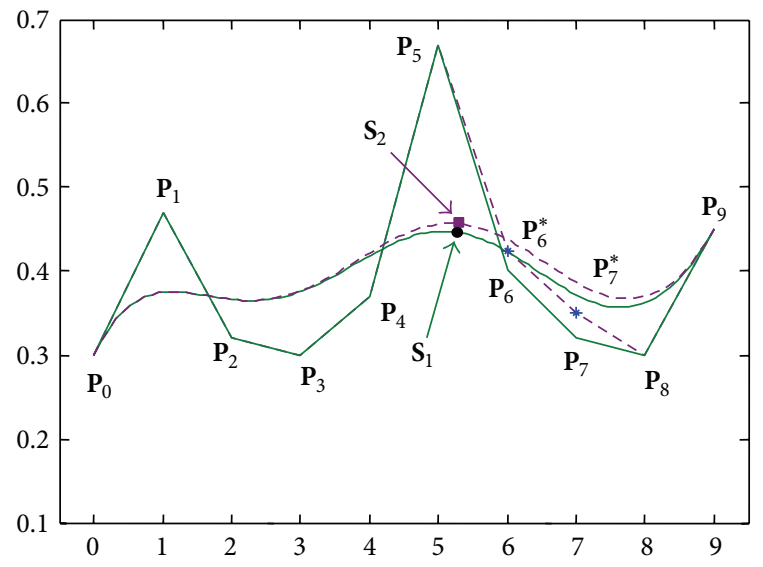

(b) Tangent vector constraint

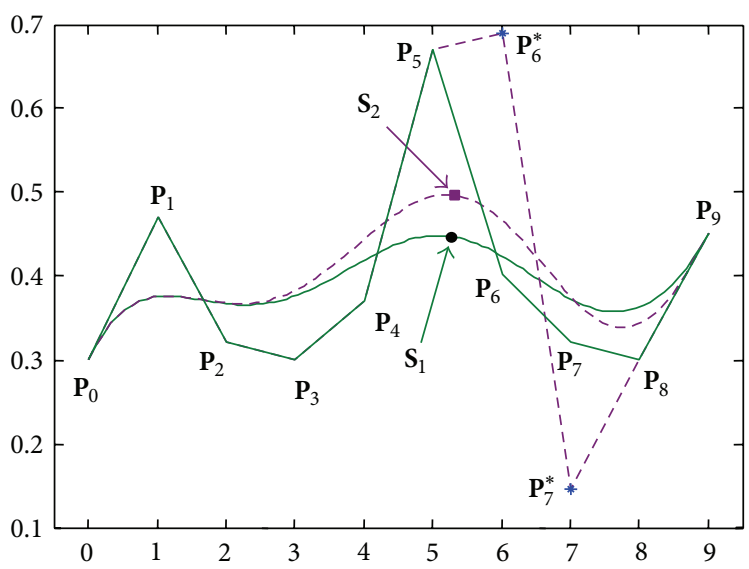

(c) Position vector and tangent vector constraints

FIgURE 7: Shape modification for $\lambda$-Bézier curves of degree nine with single target point.

shown as green solid lines and modified curves are shown as red dashed lines. Original target points $\mathbf{S}_{1}$ are black dot and modified target points $\mathbf{S}_{2}=\mathbf{p}_{2}\left(0.6 ; \lambda_{1}\right)$ are red square point. $\mathbf{P}_{i}^{*}(i=3,4)$ are modified control points, and the other control points of original curves remain unchanged.

Example 3. Given shape parameter $\lambda_{1}=1$ and control points $\left\{\mathbf{P}_{0}=(0,0.3), \mathbf{P}_{1}=(1,0.47), \mathbf{P}_{2}=(2,0.32), \mathbf{P}_{3}=\right.$ $(3,0.3), \mathbf{P}_{4}=(4,0.37), \mathbf{P}_{5}=(5,0.67), \mathbf{P}_{6}=(6,0.4), \mathbf{P}_{7}=$ $\left.(7,0.32), \mathbf{P}_{8}=(8,0.3), \mathbf{P}_{9}=(9,0.45)\right\}$, we can construct an original $\lambda$-Bézier curve of degree 9. Figure 7 illustrates the shape modification for it by constrained optimization of position and tangent vector of single target point $\mathbf{S}_{1}=$ $\mathbf{p}_{1}\left(0.6 ; \lambda_{1}\right)$, where original curves are shown as green solid lines and modified curves are shown as violet dashed lines. Original target points $\mathbf{S}_{1}$ are black dot and modified target points $\mathbf{S}_{2}=\mathbf{p}_{2}\left(0.6 ; \lambda_{1}\right)$ are violet square point. $\mathbf{P}_{i}^{*}(i=6,7)$ are modified control points and the other control points of original curves remain unchanged.

\section{Conclusions}

In this paper, we give the definition of $\lambda$-Bézier curves and discuss their properties in detail. It is shown that
$\lambda$-Bézier curves of degree $n$ with shape parameter keep many properties of the corresponding traditional Bézier curves and are more convenient than traditional ones. We can alter the shape of $\lambda$-Bézier curve by modifying the values of the shape parameter without changing its control points. Further, we investigate the shape modification of $\lambda$-Bézier curves for constrained optimization of single point constraint (including modification of shape parameter and control points). In order to modify the shape of $\lambda$-Bézier curves effectively, we obtain some explicit formulas for modifying control points and shape parameter. Three practical examples show that the method is applicable for computer aided design system. Future work will focus on studying the shape modification for $\lambda$-Bézier surfaces.

\section{Conflict of Interests}

The authors declare that there is no conflict of interests regarding the publication of this paper.

\section{Acknowledgments}

The authors are very grateful to the referees for their helpful suggestions and comments which have improved the paper. 
This work is supported by the National Natural Science Foundation of China (nos. 51305344 and 11426173). This work is also supported by the Research Fund of Department of Science and Department of Education of Shaanxi, China (no. 2013JK1029).

\section{References}

[1] G. Farin, Curves and Surfaces for CAGD: A Practical Guide, Academic Press, San Diego, Calif, USA, 5th edition, 2002.

[2] L. Piegl, "Modifying the shape of rational B-splines. Part 1: curves," Computer-Aided Design, vol. 21, no. 8, pp. 509-518, 1989.

[3] J. Sànchez-Reyes, "A simple technique for NURBS shape modification," IEEE Computer Graphics and Applications, vol. 17, no. 1, pp. 52-59, 1997.

[4] I. Juhász, "Weight-based shape modification of NURBS curves," Computer Aided Geometric Design, vol. 16, no. 5, pp. 377-383, 1999.

[5] S. M. Hu, D. W. Zhou, and J. G. Sun, "Shape modification of NURBS curves via constrained optimization," in Proceedings of the 6th International Conference on Computer Aided Design and Computer Graphics (CAD/Graphics '99), pp. 958-962, Shanghai, China, September 1999.

[6] S.-M. Hu, Y.-F. Li, T. Ju, and X. Zhu, "Modifying the shape of NURBS surfaces with geometric constraints," Computer Aided Design, vol. 33, no. 12, pp. 903-912, 2001.

[7] L. Xu, Y. J. Chen, and Y. N. Hu, "Shape modification of Bézier curves by constrained optimization," Journal of Software (China), vol. 13, no. 6, pp. 1069-1074, 2002.

[8] Q.-B. Wu and F.-H. Xia, "Shape modification of Bézier curves by constrained optimization," Journal of Zhejiang University: Science, vol. 6A, supplement 1, pp. 124-127, 2005.

[9] Z. Wang, L. Zhou, and X. Wang, "Weight-based shape modification of NURBS curves by constrained optimization," Journal of Southeast University, vol. 20, no. 4, pp. 458-462, 2004.

[10] I. Juhász and M. Hoffmann, "Constrained shape modification of cubic B-spline curves by means of knots," Computer Aided Design, vol. 36, no. 5, pp. 437-445, 2004.

[11] X. Han and Y. Ren, "Shape modification of Bezier curves by constrained optimization of position and tangent vector," Journal of Computer-Aided Design and Computer Graphics, vol. 20, no. 9, pp. 1191-1195, 2008.

[12] Q. Y. Chen and G. Z. Wang, "A class of Bézier-like curves," Computer Aided Geometric Design, vol. 20, no. 1, pp. 29-39, 2003.

[13] X.-A. Han, Y. Ma, and X. Huang, "The Cubic trigonometric Bézier curve with two shape parameters," Applied Mathematics Letters, vol. 22, no. 2, pp. 226-231, 2009.

[14] X. A. Han, Y. Ma, and X. Huang, "A novel generalization of Bézier curve and surface," Journal of Computational and Applied Mathematics, vol. 217, no. 1, pp. 180-193, 2008.

[15] L. Q. Yang and X.-M. Zeng, "Bézier curves and surfaces with shape parameter," International Journal of Computer Mathematics, vol. 86, no. 7, pp. 1253-1263, 2009.

[16] L. L. Yan and J. F. Liang, "An extension of the Bézier model," Applied Mathematics and Computation, vol. 218, no. 6, pp. 28632879, 2011.

[17] J. Chen, "Quasi-Bézier curves with shape parameters," Journal of Applied Mathematics, vol. 2013, Article ID 171392, 9 pages, 2013.
[18] X. Qin, G. Hu, N. Zhang, X. Shen, and Y. Yang, "A novel extension to the polynomial basis functions describing Bézier curves and surfaces of degree $n$ with multiple shape parameters," Applied Mathematics and Computation, vol. 223, pp. 1-16, 2013.

[19] X. Q. Qin, G. Hu, Y. Yang, and G. Wei, "Construction of PH splines based on H-Bézier curves," Applied Mathematics and Computation, vol. 238, no. 6, pp. 460-467, 2014.

[20] G. Hu, X. Ji, and L. Guo, "Quartic generalized Bézier surfaces with multiple shape parameters and its continuity conditions," Transactions of the Chinese Society for Agricultural Machinery, vol. 45, no. 5, pp. 315-321, 2014.

[21] Y. P. Zhu, X. L. Han, and S. G. Liu, "Curve construction based on four $\alpha \beta$-Bernstein-like basis functions," Journal of Computational and Applied Mathematics, vol. 273, no. 1, pp.160181, 2015. 


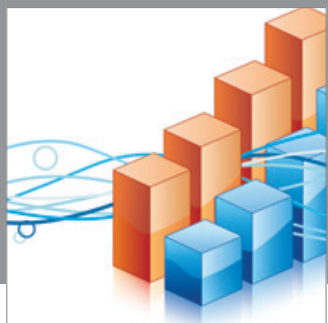

Advances in

Operations Research

mansans

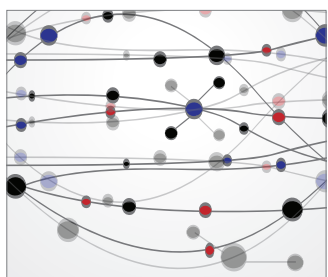

The Scientific World Journal
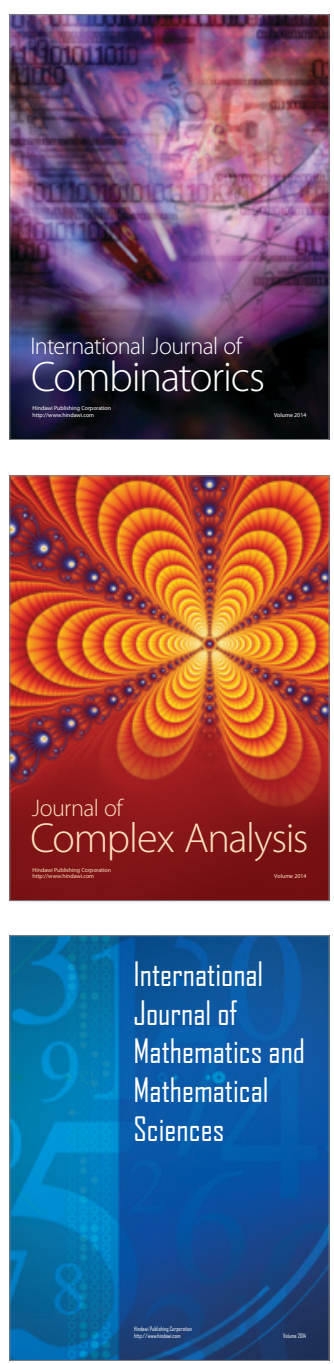
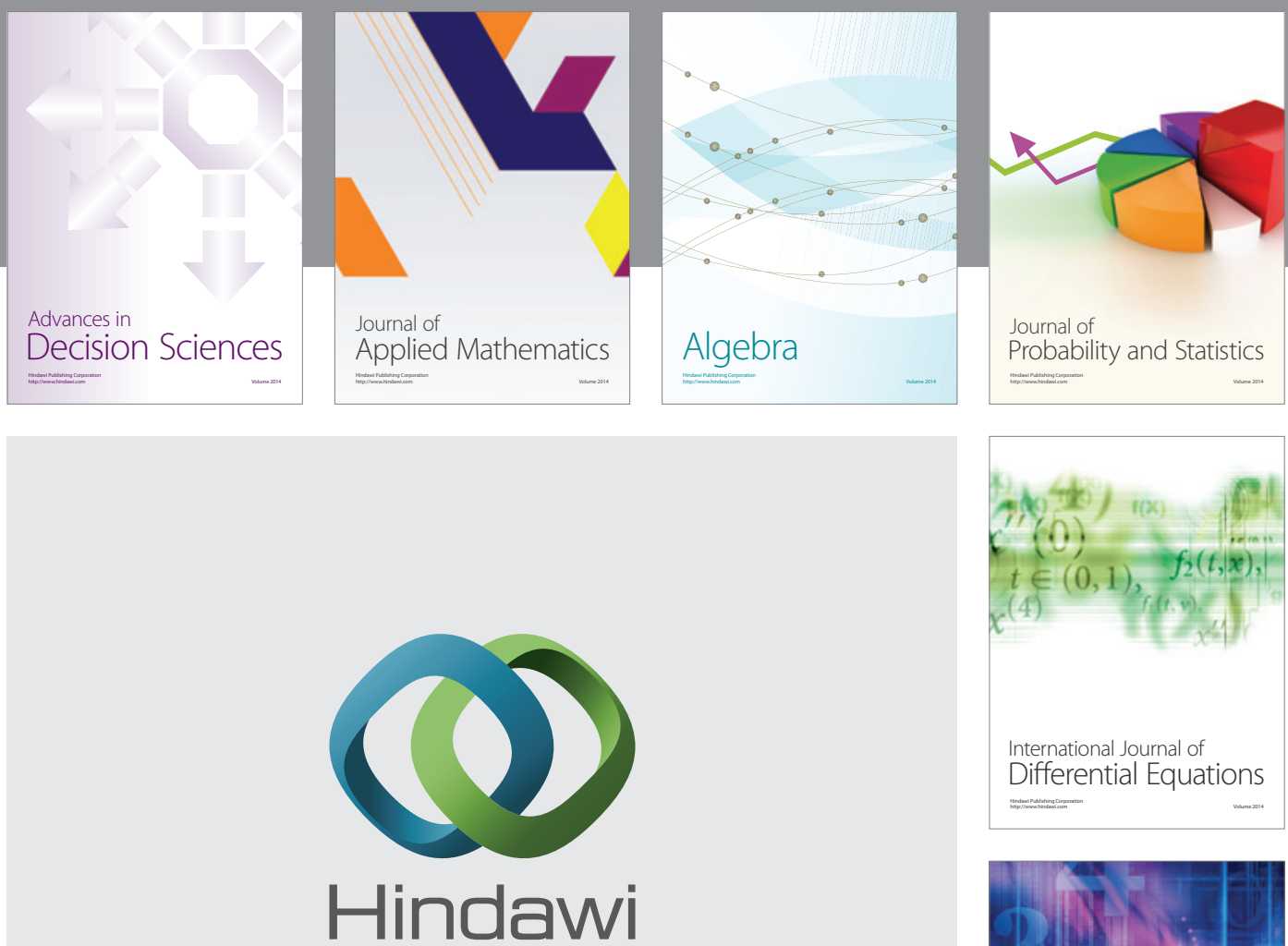

Submit your manuscripts at http://www.hindawi.com
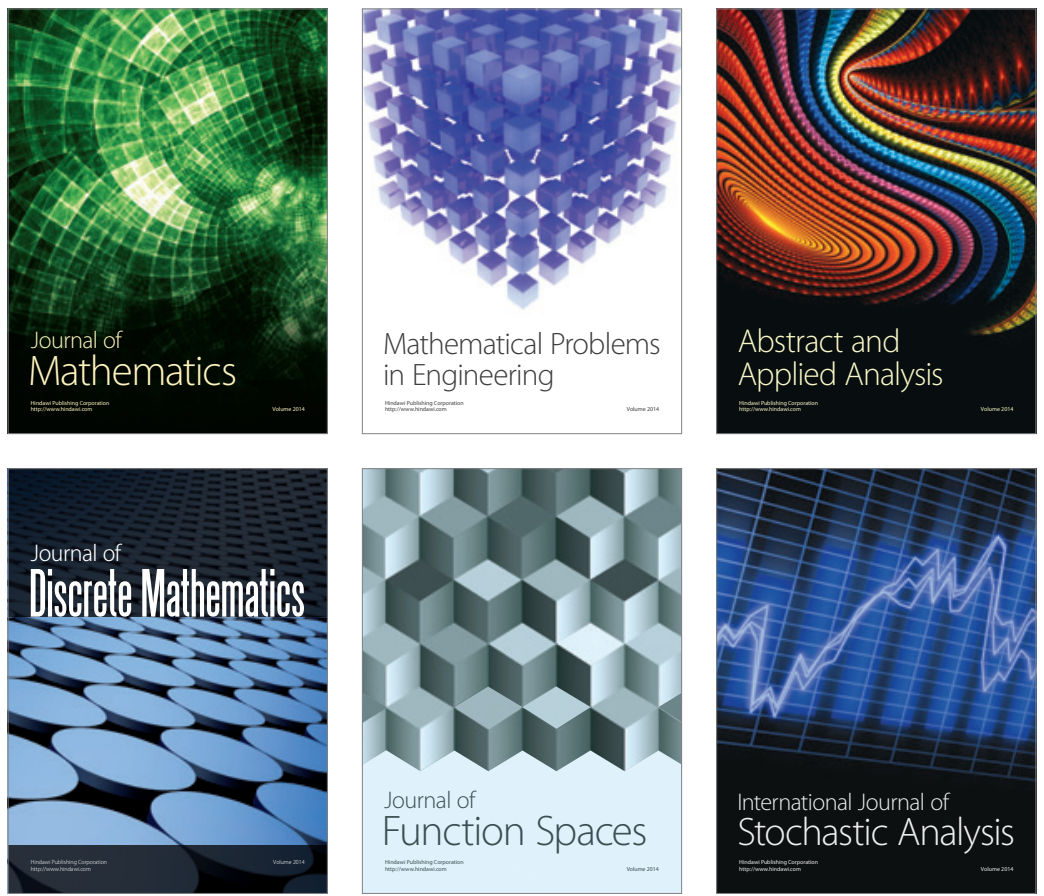

Journal of

Function Spaces

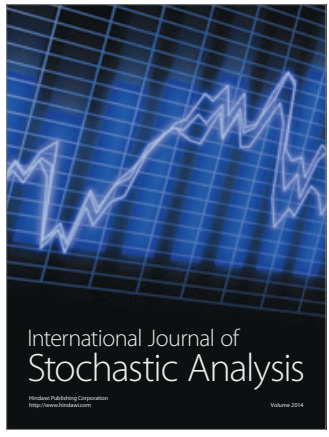

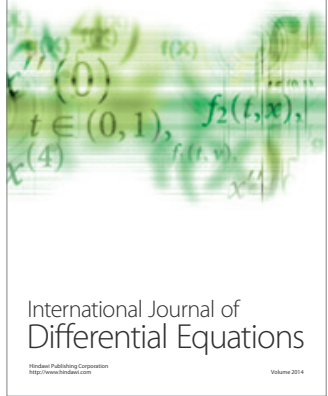
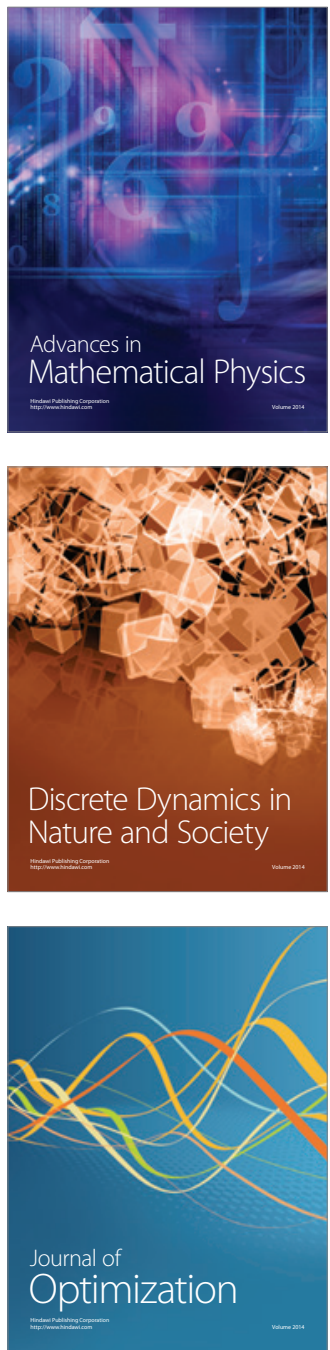